\title{
VI. Six theorems, containing the chief properties of all regular douzeave systems of music; with twelve corollaries thence deduced, showing others of their relations; and thirteen scholia, containing the temperaments of as many systems, calculated thereby. with remarks
}

\section{Mr. John Farey}

To cite this article: Mr. John Farey (1810) VI. Six theorems, containing the chief properties of all regular douzeave systems of music; with twelve corollaries thence deduced, showing others of their relations; and thirteen scholia, containing the temperaments of as many systems, calculated thereby. with remarks, Philosophical Magazine Series 1, 36:147, 39-53, DOI: $10.1080 / 14786441008563135$

To link to this article: http://dx.doi.org/10.1080/14786441008563135

Published online: 18 May 2009.

Submit your article to this journal ¿

山ll Article views: 2 
free space inside; the increase of expense on this account would not, I suppose, much exceed twelve or fifteen thousand pounds, in addition to that before -stated.

$$
\begin{aligned}
& \text { I am, sir, } \\
& \text { Your obedient humble servant, } \\
& \text { W. Caulfield LEnNox. }
\end{aligned}
$$

Bath, June 1810.

VI. Six Theorems, containing the chicf Properties of all Regular Douzeave Systems of Music; with Twelve Corollaries thence deduced, showing others of theirRelations; and Thirteen Scholia, containing the Temperaments of as muny Systems, calculated thereby. With Remarks. By Mr. John FareY.

\section{To $M r$. Tilloch.}

SIR, I AM much pleased to observe, that at length a beginning has been made, at publishing tables of the Beats in 15"; made by the 72 concords in different systems of Musical Temperament, by your new correspondent $\mathrm{Mr}$. C. J. Smyth, of Norwich, in your last Number, who will, I hope, persevere, and give us tables of many other systems, accompanied by such critical remarks, on their comparative merits and defects in practice, as he appears well qualified to make, either in your Magazine, or in the separate work which he has announced on the subject.

Some time ago, I had thoughits of preparing a work on Harmonics, perhaps as a kind of supplement to Dr.-Robert Smith's justly celebrated work ; but the prospect being now rather distant, of my being able to find leisure to complete this design, I am induced by the above paper of Mr. Smyth's, and the publication of a small work on Harmonics, by Mr. J. Marsh of Chichester, to transcribe from my papers some Theorems, showing the properties of regular douzeaves, or of such systems of twelve notes in the octave, as have all their fifths alike tempered, except, that between $\mathrm{G}$ and $\mathrm{bE}$, when there is a bearing fifth or quint wolf: but first I beg to make a few remarks.

In douzeaves, or systems of twelve nntes, there are generally 16 wolves or tempered concords, differing * from

* Wolves, taken in their general sense, are not always larger than the temperaments, but may be equal to them, as happens throughout the isotonic or equal temperament scale, and may evert be less than their respective temperaments, in some cases, as in scholia 1 and $7:$ they are, in fact, the places in the douzeave ct other defective scales, where the resulting intervals or unavoidable inequalities fall, and, as such, are very important to be known and attended to by the composers of music, to be performed in such scales. 
the temperaments proper to the six several concords re, spectively.

In regular douzeaves, none of these wolves occur, in any of the six concords, upon any of the four middlemost key-notes, viz. G, D, A or E respectively.

$C, F, b B$ and $b E$ have no wolves in their major concords (i.e. the III, V or VI upon them, respectively.)

$\mathrm{B}, * \mathrm{~F}, \mathrm{C}$ and $* \mathrm{G}$ have no wolves in their minor concords (i.e. $3 \mathrm{~d}, 4 \mathrm{th}$, or 6 th.)

Whence it follows (sée Dr. Smith's Harmonics, Plate XIX, p. 162, 2d edit.) that in major Keys, modulation can be made from $C$ by $* s$, through the keys $G, D, A$ and $E$ without false concords or wolves; but if we proceed further by $* \mathrm{~s}, \mathrm{~B}$ has a false III, $\mathrm{F}$ has its III and VI false, $* \mathrm{C}$ has its III and VI false, and $G$ has its V, III and VI false, which last chord Mr. Smy:h calls the wolf, by way of eminence, p. 450 of last volume.

And in major keys, modulation can also be made from $\mathbf{C}$ by bs, through $F, b B$, and $b E$, and no major wolves occur, (yet $b E$ has a false $4 \mathrm{th}$ ); but on proceeding further to $b A$ (or G) its V, III and VI are false, as above.

In minor Keys, modulation can be made from $\mathrm{A}$ by *s through $\dot{E}, B, * F, C$, and $* G$, without any minor wolf (yet * $G$ has a false $V t h$ ), but on proceeding further to $* D$ (or $b E$ ), its $4 \mathrm{th}, 3 \mathrm{~d}$ and 6 th are false.

And in minor keys, modulation can also be made from $A$ by bo through $D$ and $G$, without any false coucords or wolves; but on continuing thus to modulate, $C$ has a false 6 th, $F$ has its $3 \mathrm{~d}$ and $6 \mathrm{th}$ false, $\mathrm{b} B$ its $3 \mathrm{~d}$ and $6 \mathrm{th}$ false, and $\mathrm{BE}$ (or $* \mathrm{D}$ ) has its $4 \mathrm{th}, 3 \mathrm{~d}$, and 6 th false, as above.

The six following Theorems, express in terms, of the fractions $\frac{r}{s}$ and $\frac{t}{u}$ (either proper or improper) of the small intervals Schisma and most Minute* or $\Sigma$ and $\mathrm{m}$, all the temperaments and wolves of the 72 concords, in any regular douzeave; and whence, such temperaments can readily be calculated for any proposed system, or the vario $s$ properues and relations of its intervals can be discovered and computed: and by means of other theorems, the beats can be calculated from such temperaments, in terms of $\Sigma$ and $m$. In the article BEATs in the "Edin-

\footnotetext{
* See vol. xxviii, p. 142, and èngraved table, plate V.
} 


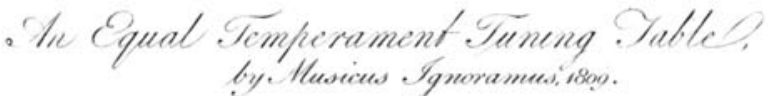

Starpened Fiftbs downwards

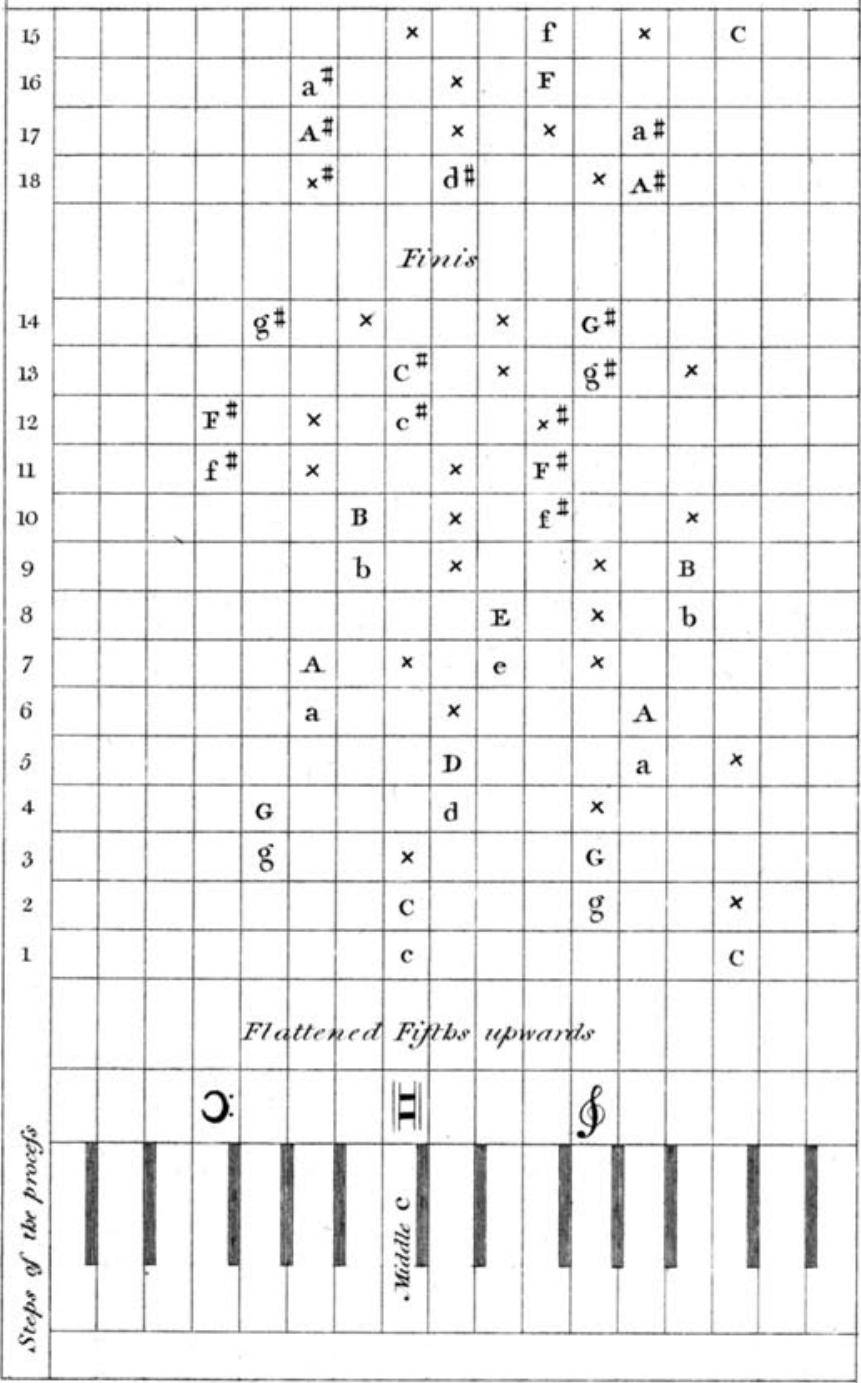


burgh Encyclopædia," such a set of Theorems will shortly be given, with examples of the use of each, as will perhaps supersede the necessify of publishing them in your Magazine.

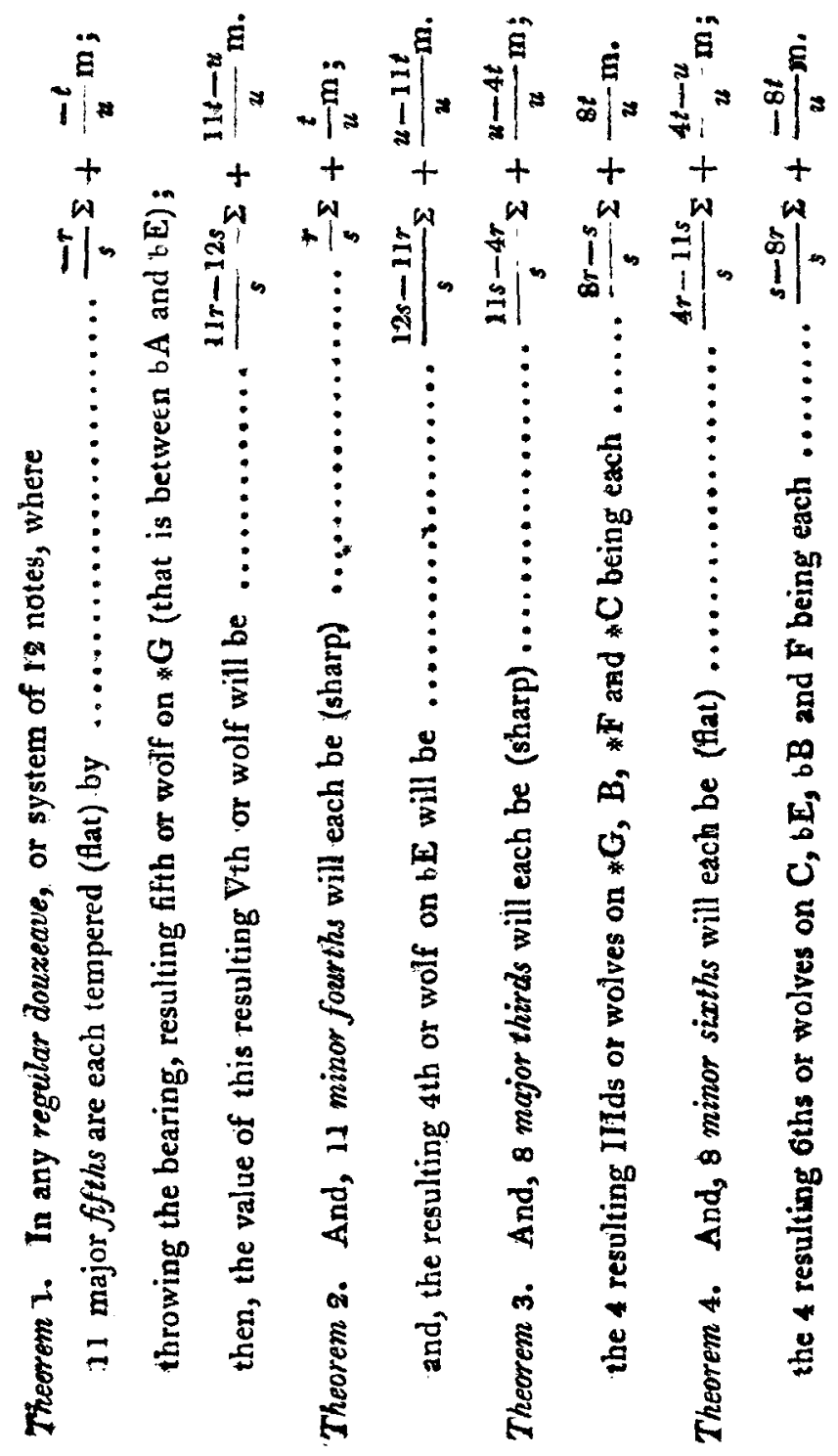




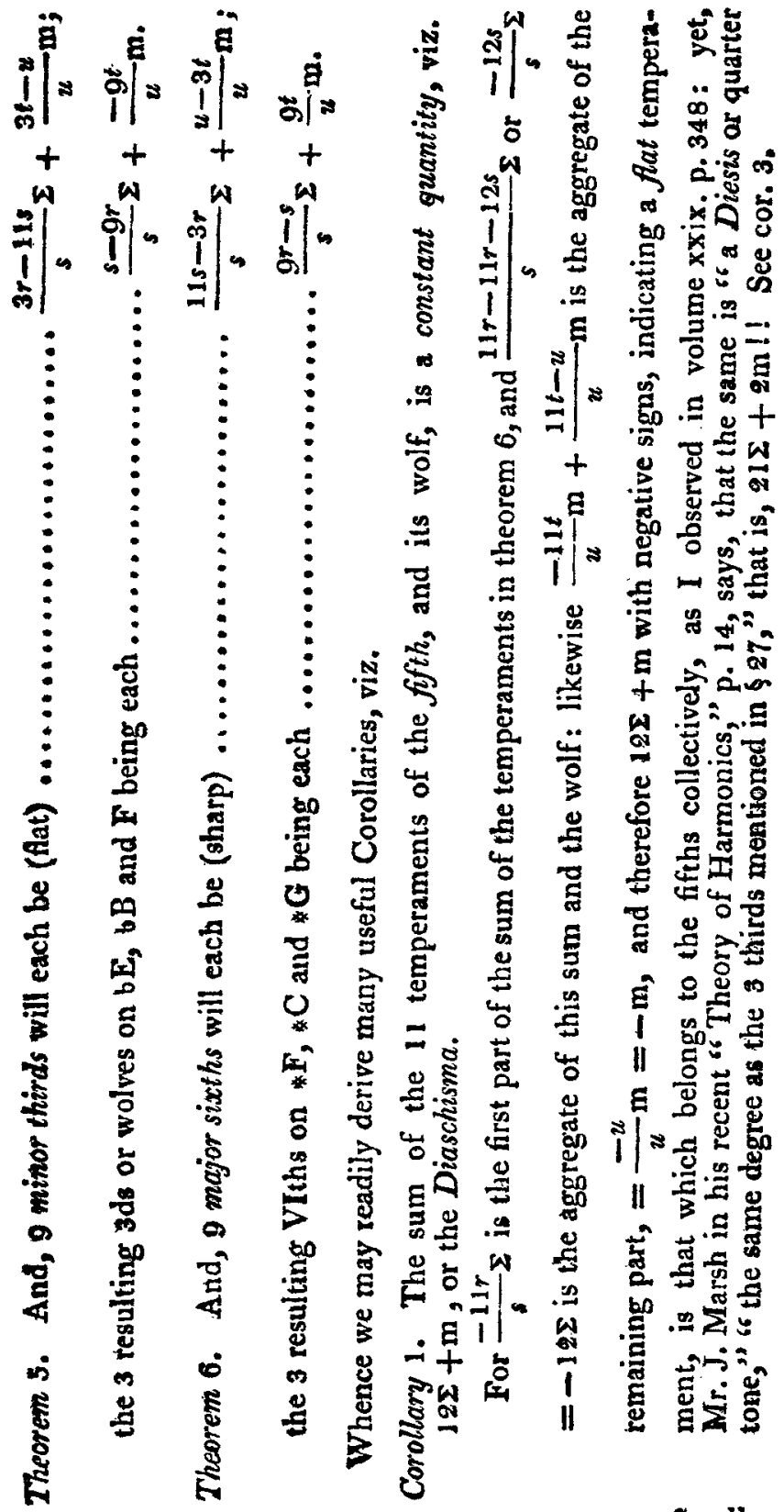

Corollary 
Corollary 2. The sum of the 11 temperaments of the minor fourth, and its wolf, is constantly $12 \Sigma+m$, as observed above.

Corollary 3. The sum of the 8 temperaments and wolves of the major thirds, is $84 \Sigma+8 \mathrm{~m}$, or four enharmonic Diesises.

Corollary 4. The sum of the 8 temperaments and 4 wolves of the minor sixths, is $-84 \Sigma-8 \mathrm{~m}$, as above.

Corollary 5. The sum of the 9 temperaments and 3 wolves of the minor thirds, is $-96 \Sigma-9 \mathrm{~m}$, or three Semitones minimum.

Corollary 6. The sum of the 9 temperaments and 3 wolves of the major sixths, is $96 \Sigma+9 \mathrm{~m}$, as above.

Corollary 7. The sum of the temperaments of the minor third and of the major third, is equal to the temperament of the fifth.

For $\frac{3 r-11 s}{s}+\frac{11 s-4 r}{s}=\frac{-r}{s}$, the first part of the temperament of the fifth, and so of the latter part.(See Dr. Smith's Harmonics, cor. 6, p. 42.)

Cornllary 8. The sum of the temperaments of the fifth and of the major sixth, is equal to the temperament of the major third.

$$
\text { For } \frac{-r}{s}+\frac{11 s-3 r}{s}=\frac{11 s-4 r}{s} \Sigma \text {; also } \frac{-t}{u}+\frac{u-3 t}{u}
$$

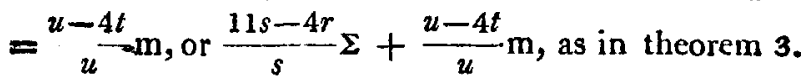
(See Dr. Smith's Harmonics, cor. 8, p. 43.)

Corollary 9. The difference between the wolf and the temperament of each of the six concords respectively, is the same, viz. $\frac{12 r-12 s}{s} \Sigma+\frac{12 t-u}{u} \mathrm{~m}$, and is what Dr. Smith, at pages 160,223 , \&c. calls the Diesis; it is the difference between adjacent flat and sharp notes, as between $D$ and $b E, A$ and $b B, \& c$, p. 163 . For $\frac{11 r-12 s}{s}-\frac{-r}{s}=\frac{12 r-12 s}{s} \Sigma$, and $\frac{11 t-u}{u}-\frac{-t}{u}$ $=\frac{12 t-u}{u} \mathrm{~m}$, in the fifths. Also, $\frac{8 r-s}{s}-\frac{11 s-4 r}{s}=$ $\frac{12 r-12 s}{s} \Sigma$, and $\frac{8 t}{u}-\frac{u-4 t}{u}=\frac{12 t-u}{u} \mathrm{~m}$, in the major third, as before, and so of all the others. 
Corollary 10. The minor Limma of Dr. Smith, p. 223, or the value of a sharp or a flat, is, $\frac{58 s-7 r}{s} \Sigma+\mathrm{f}+$ $\frac{5 u-7 t}{u} \mathrm{~m}$; or, without $\mathrm{f}^{*}$, we have $\frac{58 \cdot 1496 s-7 r}{s}+$ $\frac{5 \cdot 0136 u-7 t}{u} \mathrm{~m}$.

As is easily deduced from my theorems above, by the process in page 158, of the Harmonics. As is also the following,

Corollary 11. The major Limma of Dr. Smith is $\frac{46 s+5 r}{s} \Sigma$ $+\mathrm{f}+\frac{4 u+5 t}{u} \mathrm{~m}$; or, without $\mathrm{f}$, we have $\frac{46 \cdot 1496 s+5 r}{s} \Sigma$ $+\frac{4.0136 u+5 t}{u} \mathrm{~m}$.

Corollary 12. The mean Tone of regularly tempered systems, is $\frac{104 s-2 r}{s} \Sigma+2 \mathrm{f}+\frac{9 u-2 t}{u} \mathrm{~m}$; or, without f we have $\frac{104 \cdot 2992 s-2 r}{s} \Sigma+\frac{9 \cdot 0272 u-2 t}{u} \mathrm{~m}$.

By multiplying these general Corollaries, I should, perhaps, exceed your limits. I must therefore content myself for the present with the following, as particular applications of the theorems and corollaries above, viz.

Scholium 1. If a douzeave be required, in which the fifths should be perfect, we have by theorem $1, \frac{-r}{s}+\frac{-t}{u}=0$; which condition will be answered, if $r$ and $t$ each $=0$ and $s$ and $u$ each $= \pm 1$; which values substituted in the fifth wolf $\frac{11 r-12 s}{s} \Sigma+\frac{11 t-u}{u} \mathrm{~m}$, gives $-12 \Sigma$ $-m$ as it ought to do, by cor. 1 .

Also, in theorem $3, \frac{11 s-4 r}{s} \Sigma+\frac{u-4 t}{u} \mathrm{~m}$ gives $11 \Sigma$ $+m$ or $c$, a major comma, as the sharp temperament of each major third in this system; also in theorem 6. $\frac{11 s-3 r}{s} \Sigma+\frac{\nu-3 t}{u} \mathrm{~m}$ gives $11 \Sigma+\mathrm{m}$ or $\mathrm{c}$, ds the sharp

- By an examination of plate $V$ in your 28 th volume, and of a more extensive table of intervals, it appears, that the number of $\Sigma s$, always exceeds the number of $\mathrm{m}$ 's in ratios between those of $12.5: 1$ and $10: 1$ (those of $S$ and $\in$ ). But the major comma and its aliquot parts, most frequently occurring in temperaments, $I$ have adopted its ratio of $11: 1$, and tlus find $f=1496 x+0136 m$; from which equation, the latter ones in cor. 10,11 and 12 are obtained.

tem- 
Temperaments of different musical Systems.

temperament of the major sixths; which last temperaments prove the same as they must do, to fulfil the conditions of cor. 8 .

Though the fifth wolf is here so large, the IIIds and VIths wolves are each $\Sigma$ only.

This system approaches very near to that of Mercator, wherein the octave is divided into 53 equal parts, and where $-.035094 \Sigma$ is the flat temperament of the fifth. See M. Sauveur's general table of tempered systems, Mem. de l'Acad. 1711, 16mo. p. $\$ 16$.

Scholium 2. If a douzeave be required, in which the major thirds should be perfect, we have in theorem 3 $\frac{11 s-4 r}{s}=0$, or $11 s=4 r$, whence $\frac{r}{s}=\frac{11}{4} ;$ also $\frac{x-4 t}{u}$ $=0$, or $u=4 t$ and $\frac{t}{u}=\frac{1}{4}$; whence it appears, that the fifth is to be flattened $\frac{11}{4} \Sigma+\frac{1}{4} \mathrm{~m}$, or $\frac{1}{4} \mathrm{c}$; and, by substituting the above values in the wolf, the same appears to be $21 \bar{z}+\varepsilon \mathrm{m}$, as in cor. 3 ; and by the same in theorem 6 , we get $\frac{11}{4} \Sigma+\frac{1}{4} m$, or $\frac{1}{4} c$, the sharp temperament of the major sixth; the same with that of the fifth; see cor. 8 .

This is the Mean Tone system of Salinas, Zarlino, Aretinus, \&c. Dr. Smith's Harmonics, p. 36, 41, \&ce. wherein the adjacent flat and sharp notes are distant $21 \Sigma+2 m$, or an enharmonic Diesis, as appears by substituting the above values of $\frac{\tau}{s}$ and $\frac{t}{u}$ in cor. 9 .

It is also nearly the same with a division of the octave into 112 equal parts, (see M. Sauveur's table above quoted), wherein $-2.8290 \mathrm{\Sigma}$ is the flat temperament of the fifth; $\frac{1}{4} \mathrm{c}$ above, being $-2.751966 \mathrm{\Sigma}$.

Scholium 3. If 2 douzeave be required, in which the major sixths should be perfect, we have in theorem $6, \frac{11 s-3 r}{s}$ $=0$, or $11 s=3 r$, whence $\frac{r}{s}=\frac{11}{3}$; also $\frac{u-3 t}{u}=0$, or, $u=3 t$; whence $\frac{t}{u}=\frac{1}{3}$, and $\frac{11}{3} \Sigma+\frac{1}{3} \mathrm{~m}$, or, $\frac{1}{3} \mathrm{c}$, is here the flat temperament of the fifth: and by sub. 
substituting the same in the wolf we get $32 \Sigma+3 \mathrm{~m}$; as in corollary 6; likewise in theorem 3 , we have $\frac{11}{3} \Sigma+{ }_{3}^{1} \mathrm{~m}$, or $\frac{1}{3} \mathrm{c}$, the sharp temperament of the major third also, as is consistent with cor. 8. The IIId and Vth wolves are here each $28 \frac{1}{3} \Sigma+2 \frac{8}{3} m$. (See Harmonics, p. 42.)

This system approaches very near to a division of the octave into 19 equal parts, where $-3.6947 \mathrm{\Sigma}$ is the flat temperament of the fifth, (Dr. Smith, Harmonics, p. 158, makes it $-\frac{1}{4} c-\frac{3}{35} c=-\frac{47}{140} c$ or $-3 \cdot 6955 \Sigma) ;-\frac{1}{3} c$ above, is $-3.669288 \Sigma$. See M. Sauveur's table.

The cases of equality of temperaments between the III and VI, and between the V and VI having occurred, in schol. 1 and 3,1 proceed to

Scholium 4. If a douzeave be required, in which the major fifth shall be as much tempered fat as the major sixth is sharp; from theorems 1 and 6 we have $\frac{-r}{s}=\frac{11 s-3 r}{s}$, or $11 s=2 r ;$ whence $\frac{r}{s}=\frac{11}{2} ;$ also $\frac{-t}{u}=\frac{u-3 t}{u}$, or $u=2 t$; whence $\frac{t}{u}=\frac{1}{2}$; and $\frac{11}{2} \Sigma$ $+\frac{1}{2} m$, or $\frac{1}{2} c$, is here the temperament of the fifth : and by substituting this value in theorem 6 , we have $\frac{11}{2} \Sigma+\frac{1}{2} m$, or $\frac{1}{2} c$, for the temperament of the major sixth also. The $\mathrm{V}$ and VI wolves are here each $48 \frac{1}{2} \Sigma+4 \frac{1}{2} m$.

Scholium 5. If a douzeave be required, in which the major fifth shall be as much tempered flat as the major third is tempered flat; from theorems 1 and 3 , we have $\frac{-r}{s}=\frac{4 r-11 s}{s}$, or $5 r=11 s$; whence $\frac{r}{s}=\frac{11}{5}$; also, $\frac{-t}{u}=\frac{4 t-u}{u}$ or $5 t=u$, whence $\frac{t}{u}=\frac{1}{5} ;$ and $\frac{11}{5} \Sigma$ $+\frac{1}{5} m$, or $\frac{1}{5} c$, is here the temperament of the fifth: and by substituting this value in theorem 3 , we have $\frac{11}{5} \Sigma+\frac{1}{5} m$, or $\frac{1}{5} c$, for the flat temperament of the major 
major third also: the temperaments of the major sixths being $\frac{22}{5} \Sigma+\frac{2}{5} m$, or $\frac{2}{5} c$.

This is the system of Mr. John Holden, since me. tamorphosed into an irregular douzeave in the article Monochord, Enc. Brit. 3d edit. vol. xii. p. 240, and into a still different one, by the Rev. Mr. Hawkes. (See your xxvith volume, p. 171, and $x \times x$ th volume, p. 5.)

It also approaches near to $M$. Sauveur's system of 43 equal parts in the octave, (see his general table above referred to) wherein $-2.1177 \Sigma$ is the flat temperament of the fifth; $\frac{1}{5} \mathrm{c}$ above, being $-2.201573 \Sigma$.

One other case of this kind, viz. where the major thirds are tempered flat as much as the major sixths are tempered sharp, will be found to arise from different considerations in scholium 11 .

Scholium 6. If a douzeave be required, in which the temperament and wolf of the fifth shall be equal, we have from theorem $1,-r=11 r-12 s$, or $12 r=12 s$, whence $\frac{r}{s}=\frac{1}{1}$; also $-t=11 t-u$, or $12 t=u$, whence $\frac{t}{u}$ $=\frac{1}{12}$, and $\Sigma+\frac{1}{12} m$, or $\frac{1}{11} c$, is the flat temperament of the fifth, in this case: and which substituted in theorem 3, either for wolf or temperament, gives $7 \Sigma+\frac{2}{3} m$, or $\frac{7}{11} c$, the sharp temperament of the major third; also, in theorem 6 , gives $8 \Sigma+\frac{3}{4} m$, or $\frac{8}{11} \mathrm{c}$, the sharp temperament of the major sixth.

This is the Isotonic or Equal Temperament System of Mersennus, \&c. called by Mr. Marsh and others, though improperly, the Equal Harmony System (see scholium 10). See vol. xxix. p. 347 : see also Dr. Smith's Harmonics, p. 158 and 167 . In the latter page, however, the temperaments of the Vth, VIth and IIId are mistakenly said to be $\frac{1}{10}, \frac{7}{10}$ and $\frac{6}{10}$, instead of $\frac{1}{11}, \frac{8}{11}$ and $\frac{7}{11}$ of a comma, as they are above, very nearly.

Scholium 7. If a douzeave be required, in which the several wolves shall differ from their respective temperaments, by the least known Interval or most Minute (m): we 
have from corollary $9, \frac{12 r-12 s}{s}=0$, or $12 r=12 s a$ whence $\frac{r}{s}=\frac{1}{1}$; also, $\frac{12 t-u}{u}=-1$, whence $\frac{t}{u}=\frac{0}{1}$. By which we obtain in theorem $1,-\Sigma$ as the fat temperament of the fifths, and $-\Sigma-m$ its flat wolf: also, in theorem 3 , we get $7 \Sigma+m$ the sharp temperaments of the major thirds, and $7 \Sigma$ their wolves $;$ and by theorem 6 , we obtain $8 \Sigma+m$ the sharp temperaments of the major sixths and $8 \Sigma$ their wolves.

This is my Equal Temperament System, whose tempered fifth, and consequently all its other intervals, can be tuned on an Organ by means of perfect intervals only, viz. 54 ths $-3 \mathrm{~V}$ ths $-\mathrm{III}=\mathrm{V}-\Sigma$. (See vol. xxviii. p. 65); such tuning to be upwards from $C$ as far as $b \mathrm{~A}$ and downwards from $c$ as far as $b \mathrm{E}$, between which notes, the wolf $\Sigma+m$, will result. The beats calculated by Mr. Smyth, at page 452 of your last volume, belong in fact to this system, and not to the strict Isotonic above, but the difference in practice would be imperceptible between these two systems.

Schotium 8. If a douzeave be required, in which the ratio of the temperaments of the major thirds shall be to their wolves as $1 \frac{1}{4}$ to $3 \frac{1}{2} *$, that is, or as 5 to 14 , we have from theorem 3 . As $5: 14:: \frac{11 s-4 r}{s}: \frac{8 r-s}{u}$; whence $154 s-56 r=40 r-5 s$, or $159 s=96 r$, and $\frac{r}{s}$ $=\frac{53}{32} ;$ also, as $5: 14:: \frac{u-4 t}{u}: \frac{8 t}{u}$, whence $14 u-$ $56 t=40 t$, or $14 u=96 t$, and $\frac{t}{u}=\frac{7}{48}:$ and $-\frac{53}{32} \Sigma-$ $\frac{7}{48} \mathrm{~m}$ is the flat temperament of the fifth, which sub. stituted in the first theorem gives $\frac{199}{32} \Sigma+\frac{29}{48} m$,

* Mr. Marsh, assuming the true major third to be 48 degrees or parts, states, the tempered III to be $=49$ a, and the wolf or " extended third," (as he elsewhere calls it) to be $51 \frac{1}{2}$ parts: in the system which he most approves; 1 therefore take the excess of these above the IIId, as giving the ratio of his temperament and wolf, in order to obtain the values of his notes in my theorems. It is not however clear, that such is exactly his meaning; since, 150 being assumed as the measure of the octave, the values of the major third and of the diesis can be no other than 48.28921, \&c. and 5.132378, \&c. er very nearly in the ratios of $612 \Sigma, 197 \Sigma$ and $21 \Sigma$ : and it is not possible for 48 and 6 truly to represent the major third and diesis in such octave, or in any other. 
the sharp wolf. In theorem 3 , we get the sharp temperament of the major third $=4 \frac{3}{8} \Sigma+\frac{5}{12} \mathrm{~m}$, and its wolf $=12 \frac{1}{4} \Sigma+\frac{7}{6} \mathrm{~m}$. And in theorem 6 , the sharp temperament of the major sixth $=6 \frac{1}{32} \Sigma+\frac{9}{16} \mathrm{~m}$, and its wolf $13 \frac{29}{32} \Sigma+\frac{21}{16} \mathrm{~m}$.

This is Mr. J. Marsh's approved method of tuning a douzeave. Theory of Harmonics, page 13.

The system nearest to this of any which I remember to bave met with, is that of a division of the octave into 67 equal parts, (see M. Sauveur's table above referred to), where $-1 \cdot 76455 \Sigma$ is the flat temperament of the fifth, which here is $-1.6574 \Sigma$; and the same differs considerably from the other system recommended by Mr. Marsh page 18, which, perhaps after the example of Dr. Smith, lue has borrowed from M. Henfling without acknowledgement. See my 10 th scbolium.

By Dr. Smith's Harmonics, 2 d edit. p. 84, prop.XI, latter part of cor. 3 , "when the bases and beats (of two tempered consonances) are the same, the temperament; have ullimately* the inverse ratio of the major terms" of the perfect ratios of these consonances. Whence

Scholium 9. If a douzeave be required, wherein the fifth $\left(\frac{2}{3}\right)$ and the major third $\left(\frac{6}{3}\right)$ to the same lase shali beat equally quick, the former flat and the latter sharp; we have from theorems 1 and 3 , as $5: 3:: \frac{-r}{s}: \frac{11 s-4 r}{s}$, whence $55 s-20 r=-3 r$, or $55 s=23 r$, and $\frac{r}{s}=\frac{55}{23}$; also, $5: 3:: \frac{-t}{u}: \frac{u-4 t}{u}$, whence $5 u-20 t=-3 t$,

- The ultimate ratios are in these cases, very near to the exact ratios: thus in scholium $9, \frac{5}{23}-c$, or $2.393013 \Sigma$, results from the ultimate ratios; the true temperament being 2:393693 2 , as derived from the length of string $l$ of the Vth, in the equation $4 l-i 3=\frac{\pi}{2}$; the difference being less than $\frac{7}{10000}$ th of a $\Sigma$, an interval altogether imperceptible in practice.

Vol. 36. No, 147. July 1810. 
or $5 u=23 t$, and $\frac{t}{u}=\frac{5}{23}$; and $\frac{55}{23} \Sigma+\frac{5}{23} \mathrm{~m}$ or $\frac{5}{23} \mathrm{c}$ is the flat temperament of the fifth. In theorem 3 , we get $18-\frac{3}{23} \Sigma+\frac{17}{23} \mathrm{~m}$ the sharp temperament of the major third; and in theorem $6,20 \frac{12}{23} \Sigma+1 \frac{22}{23} \mathrm{~m}$ the sharp temperament of the major sixth.

This is the system for defective scales which Dr. Smith describes, and recommends, pages $219,215,189,213$, and 212: and of which MFr. Atwood has (but without ackrowledgement) given the lengths of strings in his "Rectilinear Motion, \&c."

A system, wherein the octave is divided into 74 equal parts, to be found in M. Sauveur's table, and where the temperament is $V-2.3838 \Sigma$, differs but very little from $V-2 \cdot 393012 \mathrm{E}$ in this system.

Scholium 10. If a douzeave be required, wherein the fifth $\left(\frac{3}{3}\right)$ and the major sixth $\left(\frac{3}{5}\right)$ shall beat equally quick, the former flat and the latter sharp: we have from theorems 1 and 6 , as $5: 3:: \frac{r}{s}: \frac{11 s-3 r}{s}$, whence $55 s-15 r=3 r$, or $55 s=18 r$ and $\frac{r}{s}=\frac{55}{18} ;$ also, as $5: 3:: \frac{t}{u}: \frac{u-3 t}{u}$, whence $5 u-15 t=3 t$ or $5 u=18 t$ and $\frac{t}{u}=\frac{5}{18}$; and $3 \frac{1}{18} \Sigma+\frac{5}{18} \mathrm{~m}$ or $\frac{5}{18} \mathrm{c}$ is the flat temperament of the fifth: which in theorem 3 , gives $-\frac{11}{9} \Sigma-\frac{1}{9} \mathrm{~m}$ or $\frac{1}{9} \mathrm{c}$, for the flat temperament of the major third: and in theorem 6 , gives $\frac{11}{6} \Sigma+$ $\frac{1}{6} \mathrm{~m}$ or $\frac{1}{6} \mathrm{c}$, the sharp temperament of the sixth.

This is the famous System of Equal Harmony in 3 octaves, invented by Dr. Robert Smith. See his. Harmonics, pages 216, 191, 206, 212, 214, \&c. And differs but little from $M$. Henfling's system, (Mem. de l'Acad. 1711, 16mo, p. 408), wherein the octave is divided into 50 equal parts, as Dr. Sinith shows in his Harmonics, p. 1.57, and states its fifth to 
Temperaments of different musical Systems.

be flattened $-\frac{1}{4} c-\frac{1}{37} c$ or $-\frac{41}{148} c$, which is $-3.04966 \Sigma$; or more correctly it is $-3.04811 \Sigma ;-\frac{5}{18} \mathrm{c}$ being $-3 \cdot 05774 \Sigma$ in the system of this scholium.

Scholium 11. If a douzeave be required, wherein the major third ( $\left.\frac{4}{5}\right)$ and the major sixih $\left(\frac{3}{5}\right)$ shall beat equally quick, the former sharp and the latter flat; we have from theorems 3 and 6 , as $5: 5:: \frac{11 s-4 r}{s}$ : $\frac{3 r-11 s}{s}$, whence $11 s-4 r=3 r-11 s$ or $22 s=7 r$, and $\frac{r}{s}=\frac{22}{7}$; also, as $5: 5:: \frac{u-4 t}{u}: \frac{3 t-u}{u}$, whence $u-4 t=3 t-u$ or $2 u=7 t$ and $\frac{t}{u}=\frac{2}{7} ;$ and $\frac{22}{7} \Sigma$ $+\frac{2}{7} \mathrm{~m}$ or $\frac{2}{7} \mathrm{c}$ is the temperament of the fifth. Which in theorem 9 , gives $-\frac{11}{7} \Sigma-\frac{1}{7} \mathrm{~m}$, or $\frac{1}{7} \mathrm{c}$, the flat temperament of the major third; and in theorem 6 , gives $\frac{11}{7} \Sigma+\frac{1}{7} m$, or $\frac{1}{7}-c$, the sharp temperament of the major sixth*.

This is the system which Dr. Smith barely mentions at page 220, on account of its differing so little from equal harmony, in my last scholium; wherein $\frac{5}{18}$ or $\frac{35}{126} \mathrm{c}$ is the temperament of the fifth, which here is $\frac{2}{7}$, or $\frac{36}{126}$; the difference being only the $\frac{1}{126}$ th part of a major comma or $.087364 \Sigma$ : also $\frac{1}{9}$ or $\frac{7}{63}$, and $\frac{1}{7}$ or $\frac{9}{63}$, have a difference of $\frac{2}{63} \mathrm{c}$, or $34946 \Sigma$, but little more considerable, in the major thirds; and $\frac{1}{6}$ or $\frac{7}{42}$, and $\frac{1}{7}$ or $\frac{6}{42}$ have a difference of $\frac{1}{42} c$, or $\cdot 26209 \mathrm{\Sigma}$ in the major sixths.

This system differs more from that of $M$. Henfling;

- Mentioned by Dr, Robison, Sup. Enc, Brit, Sd edit, ii, 662.

D 9 
(see Sauveur's table above referred to) than the last, since $\frac{2}{7} \mathrm{c}=-3.145 \mathrm{l} \Sigma$, and in his system $-3.04811 \Sigma$ is the temperament of the fifth.

Scholium 12. If a donzeave be required, whercin the mean Tone thereof is to its major Limma as 5 lo 3 , we have from corollaries 11 and 12 , as $5: 3:: \frac{104 \cdot 2092 s-2 r}{s}$ : $\frac{46 \cdot 1496 s+5 r}{s}$, whence $230 \cdot 7450 s+25 r=312 \cdot 8976 s-$ $6 r ;$ or $82 \cdot 1496 s=31 r$, and $\frac{r}{s}=\frac{82 \cdot 1496}{31} ;$; also as 5 : $3:: \frac{9^{\circ} 0272 u-2 t}{u}: \frac{4.0136 u+5 t}{u}$, whence $20.0680 u+$ $25 t=27 \cdot 0816 u-6 t$, or $7 \cdot 013 \hat{b} u=-31 t$ and $\frac{t}{u}=$ $\frac{-7 \cdot 0136}{31}$; and $-\frac{82 \cdot 1496}{31} \Sigma^{\circ}-\frac{7 \cdot 0136}{31} \mathrm{~m}$ is the flat temperament of the fifth.

This is the syistem of $M$. Huygens. (See Dr. Smith's Harmonics, pages 158, 168, 121, 208, 224, \&c.) whose temperament of the fifth, as calculated by Dr. Smith, is $-\frac{1}{4} c+\frac{1}{110} c$ or $-\frac{53}{220} \mathrm{c}$, = $2.6519 \mathrm{\Sigma}$; mine above being about $2.6518 \mathrm{\Sigma}$.

The octave here is supposed to be divided into 31 equal parts. See M. Sauveur's table ; Mr. Ambrose Warren, in 1725, pretended to the discovery of this sysiem. Sce Monthly Magazine, vol, sxix. page413.

Scholium 13. If a dnuzeave be required, wherein the mean Tone thereof is to its major Limma as 9 to 5 , we have from corollaries 11 and 12 , as $9: 5:: \frac{104 \cdot 2992 s-2 r}{s}$ : $\frac{46 \cdot 1496 s+5 r}{s}$, whence $415 \cdot 3464 s+45 r=521 \cdot 4960 s-$ $10 r$, or $55 r=106 \cdot 1496 s$, and $\frac{r}{s}=\frac{106 \cdot 1496}{55}$; also as $9: 5:: \frac{9^{\circ} 0272 u-2 t}{u}: \frac{4 \cdot 0136 u+5 t}{u}$, whence $36 \cdot 1224 u+45 t=45 \cdot 1360 u-10 t$, or $9 \cdot 0136 u=55 t$ and $\frac{t}{u}=\frac{9.0136}{55} ;$ and $-\frac{106.1496}{55} \Sigma-\frac{90136}{55} \mathrm{~m}$ is the flat ten perament of the fifth, $=-1.9313 \Sigma$. 
This system answers to a division of the octave into 55. equal parts, and according to the papers of M. Sauveur in the Memoirs of the Parisian Academy, for 1707 and 1711, it was the system used by the Musicians of Paris at or previous to that time. See his general table of tempered systems above referred to.

$$
\begin{array}{r}
\text { I am, sir, } \\
\text { Westminster, July 11, 1.810. Your obedient humble servant, } \\
\text { J. FAREY. }
\end{array}
$$

VII. Report on the Memoirs presented to the Society of Pharmacy at Paris, in consequence of the Prizes offered in the Year 1809. Fartacted by $M$. Bouillon LAGRANGE from the full Report drawn up by Messrs. Nachet, Derosne, and Vallee.

$\mathrm{O}_{1}$ F uine memoirs sent to the Society, two have particularly fixed the attention of the committee. They were written in answer to the following question:

"To prepare the acetate of potash, so as to obtain it white and saturated, without employing radical vinegar, and without having recourse to fusion;- - to point out which of the two, the acid or the alkali, gives it the colouring principle."

The first memoir, with the motto Ex cognitis incognita, is written with great precision.

The author, after baving ascertained the advantage which would result from obtaining this salt in all its purity by a simple and œconomical process, begins by inquiring from whence the colouring principle arises: It cannot, he says, be owing to the alkali, when it is considered that the fusion of the acetate of potash renders it insoluble, and that the heat requisite for this fusion is not so.strong as that which is necessary for the preparation of any given potash; and on the other.hand, it cannot be essential to the acetic acid, when radical vinegar is capable of instantly furnishing a colourless salt. Consequently, this colouring principle must be a foreign substance contained in common vinegar, and which may be introduced into it in distillation. But this same principle is less volatile than the acetic acid, since distilled vinegar leaves a residue of it if we evaporate it a second time: it is not very soluble by itself, and it cannot be dissolved except by the addition of acetic acid since it is precipitated, at least in part, when we saturate the latter by potash: and finally, it has been ascertained that 